Radial and Nonradial Pulsations as Probes of Stellar Physics

ASP Conference Series, Vol. 259, 2002

C. Aerts, T.R. Bedding, \& J. Christensen-Dalsgaard, eds.

\title{
GSC 4778-00324, a New Small-Amplitude Multiperiodic $\delta$ Scuti Star ${ }^{1}$
}

\author{
P. Lampens ${ }^{1}$, P. Van Cauteren ${ }^{2}$, P. Niarchos ${ }^{3}$, K. Gazeas ${ }^{3}$,

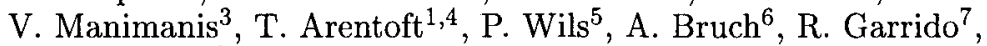 \\ R. Shobbrook ${ }^{8}$ \\ ${ }^{1}$ Koninklijke Sterrenwacht van België, Ringlaan 3, Brussels, Belgium \\ ${ }^{2}$ Beersel Hills Observatory and VVS, Belgium \\ ${ }^{3}$ Department of Astrophysics, Astronomy and Mechanics, University of \\ Athens, GR-15784 Zografos, Athens, Greece \\ ${ }^{4}$ Vrije Universiteit Brussel, Pleinlaan 2, 1050 Brussels, Belgium \\ ${ }^{5}$ Vereniging Voor Sterrenkunde (VVS), Belgium \\ ${ }^{6}$ Laboratório Nacional de Astrofísica, C.P. 21, 37500-000 Itajubá, Brazil \\ ${ }^{7}$ Instituto de Astrofísica de Andalucía, CSIC, Ap. 3004, Granada, Spain \\ ${ }^{8}$ Research School of Astronomy and Astrophysics, Australian National \\ University, Weston Creek PO, ACT 2611, Australia
}

\section{Introduction, data, analysis}

A careful analysis of the light curves of comparison stars to the intermediate amplitude $\delta$ Scuti star V1162 Ori observed in 1999/2000 within an international campaign (Arentoft et al., 2001) revealed two new variable stars: GSC 477800324 , a small-amplitude $\delta$ Scuti star, and GSC 4778-00001, an eclipsing variable (see http://www.lunarpages.com/bho for a map of the field). Here, we will concentrate on the first of these.

Since the colour indices (Handler, 2000) put GSC 4778-00324 right in the middle of the $\delta$ Scuti instability strip, we decided to monitor its variability (Van Cauteren \& Lampens, 2000) taking as much advantage as possible from the international campaign on V1162 Ori and adopting the same comparison stars. The new observations were performed between October 1999 and March 2001, mainly from the Greek and Belgian sites, making use of a standard $V$ filter. We collected and used 2866 data points for GSC 4778-00324 $(V=10.26$; $b-y=0.20)$.

The frequency analysis was performed in three steps: (a) on the (Northern) winter 1999/2000 data set, (b) on the (Northern) winter 2000/2001 data set, and (c) on the complete data set, with weights assigned proportional to the inverse square of the residuals on a night-to-night basis (Arentoft et al., 1998). Our results are displayed in Table 1. In each set up to three frequencies could be identified. The two most dominant frequencies are common to each analysis and have $\mathrm{S} / \mathrm{N}$ well above 4 . We are not sure about the third frequency found in the complete data string although it has $\mathrm{S} / \mathrm{N}>4$. After a simultaneous prewhitening with two frequencies, the remaining standard deviation is $6.9 \mathrm{mmag}$, in good agreement with the nightly rms values of the data ranging from 3 to $10 \mathrm{mmag}$.

In conclusion, we confirm that GSC 4778-00324 is a multiperiodic $\delta$ Scuti star. We have identified at least two, possibly three, frequencies in the range 
Table 1. Yearly frequency analysis of the new $\delta$ Scuti star

\begin{tabular}{|ccccccc|}
\hline Season & Nr of data & Ident. & $\begin{array}{c}\text { Frequency } \\
\mathrm{c} \mathrm{d}^{-1}\end{array}$ & $\begin{array}{c}\text { Ampl. } \\
\mathrm{mag}\end{array}$ & $\begin{array}{c}\text { Phase } \\
2 \pi \mathrm{rad}\end{array}$ & $\mathrm{S} / \mathrm{N}$ \\
\hline $1999-2000$ & \multirow{2}{*}{1393} & $\mathrm{f}_{1}$ & 12.2802 & 0.0046 & 0.979 & 5.1 \\
& & $\mathrm{f}_{2}$ & 16.6176 & 0.0042 & 0.705 & 6.8 \\
& & $f_{3}$ & 10.8231 & 0.0022 & 0.715 & $<4$ \\
\hline \multirow{2}{*}{$2000-2001$} & \multirow{2}{*}{1473} & $\mathrm{f}_{1}$ & 12.2794 & 0.0043 & 0.481 & 7.0 \\
& & $\mathrm{f}_{2}$ & 16.6216 & 0.0040 & 0.674 & 6.9 \\
& & $f_{3}$ & 13.2564 & 0.0032 & 0.706 & 5.3 \\
\hline $1999-2001$ & \multirow{2}{*}{2866} & $\mathrm{f}_{1}$ & 12.2810 & 0.0041 & 0.124 & 8.0 \\
& & $\mathrm{f}_{2}$ & 16.6196 & 0.0040 & 0.070 & 8.7 \\
& & $f_{3}$ & 12.5903 & 0.0024 & 0.302 & 4.6 \\
\hline
\end{tabular}

10-15 $\mathrm{c} \mathrm{d}^{-1}$ on the basis of new multisite data, with amplitudes well below 0.01 mag. A more detailed report will be submitted to Communications in Asteroseismology.

\section{Acknowledgements}

This project was co-supported by the Flemish Ministry for Foreign Policy, European Affairs, Science and Technology (BIL 98/11/52), the National Research Foundation of South Africa and by the Belgian Fund for Scientific Research Flanders (G.0265.97). RS, Honorary Visiting Fellow at the Australian National University, acknowledges support from the Mount Stromlo and Siding Spring Observatories.

\section{References}

Arentoft, T. et al. 1998, A\&A, 338, 909

Arentoft, T. et al. 2001, A\&A, 374, 1056

Handler, G. 2000, IBVS, 4817

Van Cauteren, P., \& Lampens, P. 2000, IBVS, 4849 